\title{
Molecular characterization of adenoviruses from children presenting with acute respiratory disease in Uberlândia, Minas Gerais, Brazil, and detection of an isolate genetically related to feline adenovirus
}

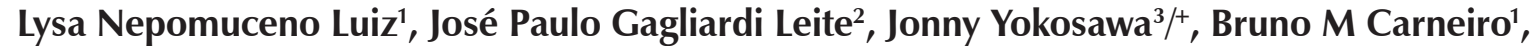 \\ Edson Pereira Filho' ${ }^{2}$, Thelma Fátima de Mattos Oliveira', Guilherme Ramos Oliveira e Freitas ${ }^{1}$, \\ Lourenço Faria Costa ${ }^{1}$, Nayhanne Tizzo de Paula ${ }^{1}$, Hélio Lopes da Silveira ${ }^{4}$, \\ Júlio César Nepomuceno ${ }^{5}$, Divina Aparecida Oliveira Queiróz ${ }^{1}$
}

\author{
${ }^{1}$ Laboratório de Virologia, Instituto de Ciências Biomédicas ${ }^{4}$ Faculdade de Medicina ${ }^{5}$ Laboratório de Citogenética e Mutagênese, \\ Instituto de Genética e Bioquímica, Universidade Federal de Uberlândia, Uberlândia, MG, Brasil \\ ${ }^{2}$ Laboratório de Virologia Comparada e Ambiental, Instituto Oswaldo Cruz-Fiocruz, Rio de Janeiro, RJ, Brasil \\ ${ }^{3}$ Division of Viral Hepatitis, Centers for Disease Control and Prevention, Atlanta, GA, USA
}

\begin{abstract}
Human adenoviruses (HAdV) are a major cause of acute respiratory diseases (ARD), gastroenteritis, conjunctivitis and urinary infections. Between November 2000-April 2007, a total of 468 nasopharyngeal aspirate samples were collected from children with ARD at the Clinics Hospital of Uberlândia. These samples were tested by immunofluorescence assay (IFA) and 3\% (14/468) tested positive for the presence of HAdV. By performing polymerase chain reaction (PCR) to detect HAdV DNA in samples that tested negative or inconclusive for all viruses identifiable by IFA (respiratory syncytial virus, parainfluenza viruses 1, 2 and 3, influenza viruses $A$ and B and HAdV), as well as negative for rhinoviruses by reverse transcription-PCR, additional 19 cases were detected, for a total of 33 (7.1\%) $H A d V$-positive samples. Nucleotide sequences of 13 HAdV samples were analyzed, revealing that they belonged to species B, C and E. Further analyses showed that species C (HAdV-2) was the most prevalent among the sequenced samples. To our knowledge, this is the first report describing the presence of HAdV-4 in Brazil. We also detected an isolate that was $100 \%$ identical to a part of the feline adenovirus hexon gene sequence.
\end{abstract}

Key words: adenovirus - acute respiratory disease - children

Acute respiratory disease (ARD) is one of the major causes of morbidity and mortality among children under the age of five, especially in developing countries (Williams et al. 2002). Approximately 5\% of these cases are associated with adenoviruses (HAdV) (Brandt et al. 1969). Furthermore, HAdV infections could account for severe lower respiratory infections in children (Murtagh et al. 2009).

HAdVs are non-enveloped, double-stranded DNA viruses with a viral particle size ranging from $70-90 \mathrm{~nm}$ in diameter with icosahedral symmetry. These viruses belong to the Adenoviridae family, which consists of 54 serotypes (HAdV-1-54) and are classified into seven species (A-G) based on their physicochemical, biological and genetic characteristics (Horwitz 2001, Jones et al. 2007, Ishiko \& Aoki 2009, Walsh et al. 2009).

In addition to the determination of the etiological agent, the characterization of $\mathrm{AdV}$ in an infection is of important clinical interest because specific sero-

Financial support: FAPEMIG, CNPq, IOC-FIOCRUZ, UFU

+Corresponding author: j.yokosawa@uol.com.br

Received 25 October 2009

Accepted 27 April 2010 types are frequently associated with the manifestation and severity of the disease (Hong et al. 2001). Indeed, HAdV-14 has recently emerged and has rapidly become a highly prevalent strain in regions of the United States, causing substantially more severe illnesses (Gray \& Chorazy 2009, Lewis et al. 2009, Tate et al. 2009). In general, neutralisation or heamagglutination inhibition assays are used for HAdV serotyping (Swenson et al. 2003). However, serotyping can be done using immunofluorescence assay (IF) with serotype-specific monoclonal antibodies (Wood et al. 1997), genus-specific polymerase chain reaction (PCR) combined with restriction endonuclease digestion (PCR-restriction fragment length polymorphism) analysis (Allard et al. 2001) and sequence analysis (Sarantis et al. 2004).

Studies conducted in Brazil reinforce the importance of HAdVs in ARD cases and they have been found as the second most frequent virus detected in nasopharyngeal aspirates (NPA) from children with ARDs in the cities of São Paulo (Moura et al. 2007) and Rio de Janeiro (Nascimento et al. 1991), located in South-eastern Brazil and Porto Alegre (Straliotto et al. 2002), located in Southern Brazil.

The main objectives of the present paper were to detect HAdV in NPA specimens from children under the age of five suffering from an ARD and to determine the HAdV serotypes that are circulating in Uberlândia, state of Minas Gerais, in South-eastern Brazil. 
Between November 2000-April 2007, 468 NPA specimens were collected at the Clinics Hospital, Federal University of Uberlândia (UFU), and processed according to Oliveira et al. (2008) at the Laboratory of Virology at UFU. This project was approved by the UFU Ethical and Research Council.

Due to the limited amount of specimen that could be collected from each child, the initial screening was carried out by IF using the Respiratory Panel I Viral Screening and Identification $\mathrm{kit}^{\circledR}$ (Chemicon International, Millipore, Temecula, CA) according to the manufacturer's instructions. This kit was used for the detection of respiratory syncytial virus (RSV), parainfluenza viruses 1, 2 and 3 (PIV 1, 2 and 3), influenza viruses A and B (Flu A and B) and HAdV. We followed the classification of results (positive, negative or inconclusive) established by Queiróz et al. (2002). RNA was extracted from IFnegative/inconclusive samples with Trizol ${ }^{\mathbb{}}$ (Invitrogen Corp, Carlsbad, CA), following the manufacturer's instructions. Reverse transcription-PCR (RT-PCR) was used to detect rhinovirus RNA as previously reported (Costa et al. 2006). The remainder of each specimen was used for the detection of HAdV.

Two rounds of detection PCR (Araújo et al. 2001) were used to amplify a $107 \mathrm{bp}$ segment of the HAdV hexon gene from samples that tested negative/inconclusive by IF and also tested negative for rhinovirus by RT-PCR. The only modification made in the protocol was the addition of one deoxyribonucleotide residue in the forward primer (5'- TGA CTT TTG AGG TGG ATc CCA TGG - $3^{\prime}$ ). Standard precautions were followed in all procedures to avoid cross-contamination.

In order to determine the serotypes of HAdV-positive specimens (detected by IF or PCR), we used a characterization PCR assay (Allard et al. 2001) to amplify a $301 \mathrm{bp}$ portion of the hexon gene and the amplicons were subsequently sequenced. The specimens that did not produce amplicons were inoculated into A-549 cells to increase the number of virus particles (Rosete et al. 2008). DNA was extracted from cell scrapes and retested using the characterization PCR assay.

PCR amplicons of the expected size (301 bp) were purified using the QIAquick PCR purification $\mathrm{kit}^{\mathbb{B}}$ (QIAGEN). Both strands were sequenced using the ABI Prism Big Dye Terminator Cycle Sequencing Ready Reaction $\mathrm{Kit}^{\circledR}$ (PE Applied Biosystems, Foster City, CA, USA). Sequence editing and analysis were performed using BioEdit (Hall 1999) and MEGA 3.1 by the neighbour-joining method (Kumar et al. 2004), respectively.

Out of 468 samples tested by IF, 3\% (14/468) were positive for HAdV. In addition, among the 126 specimens that tested negative for respiratory viruses in the IF assay (RSV, PIV 1, 2 and 3, Flu A and B) and for rhinoviruses using RT-PCR (Costa et al. 2006), 19 positive samples were obtained using the PCR detection assay. This increased the total rate of HAdV-positive specimens to $7.1 \%(33 / 468)$. This rate $(7.1 \%)$ is similar to the rate $(6 \%)$ in Southern Brazil described by Straliotto et al. (2002). However, other studies carried out in Brazil reported prevalence rates that varied from a slightly higher rate $(9.9 \%)$ for cases in the Northeast (de Arruda et al. $1991)$, to a slightly lower rate $(4.6 \%)$ in the Southeast (Vieira et al. 2001) and a much lower rate (0.8\%) in the South (Tsuchiya et al. 2005).

Although IF is a more affordable and rapid diagnostic method, PCR-based assays are also of great importance due to their higher sensitivity in the detection of HAdV (Larrañaga et al. 2007). In this study, PCR allowed us to increase the detection rate from $3 \%$, based only on the IF assay, to $7.1 \%$ by combining IF and PCR results.

The seasonal distribution pattern obtained in this study revealed that HAdV cases occurred in all seasons, similar to the results reported by Cabello et al. (2006). However, in our study approximately half of the cases were detected in the autumn. The high number of cases observed during this season may be due to a higher number of patients with ARD attending the hospital. The median age of the patients was 13 months old (range: 3-60 months) and upper respiratory tract infection was verified in approximately $66.7 \%(8 / 12)$ of the cases.

Of the total $33 \mathrm{HAdV}$ positive specimens, 31 NPAs were tested using the characterization PCR assay (2 of the samples lacked enough material to be tested). Ten of these samples tested positive and were subsequently sequenced. The remaining 21 samples were inoculated into cell culture (A-549) and their DNA was extracted from cell scrapes. From these, six additional PCR-positive samples were obtained. However, due to the insufficient amount of PCR products, only three of them were sequenced, providing a total of 13 sequenced samples. Some factors may have contributed to the limited number of specimens that tested positive using the PCR method described by Allard et al. (2001). NPAs were collected within five days after the onset of symptoms and it is possible that some of the specimens collected on the 5th day contained a low number of infected cells and/ or had a low virus titre. In addition, we used freshly collected cells in the IF assay and stored specimens for PCR and inoculation of cell culture. Moreover, detection PCR was performed in two rounds (nested PCR), while characterization PCR was carried out in one round. Lastly, the use of fresh samples would probably help to increase the number of positive specimens for characterization, but performing the test daily would be impractical and cumbersome. Identification of the nucleotide sequences from a highly conserved region among various serotypes that allow for characterization for the development of a two-round PCR assay would increase the sensitivity of detection and allow for the characterization of more specimens. However, this task is very difficult and is a subject for another study.

Through a BLAST (http://blast.ncbi.nlm.nih.gov) search with sequences obtained in this study, we determined different HAdV genotypes: eight sequences were highly identical to HAdV of species C (7 HAdV-2 and $1 \mathrm{HAdV}-1$ ), four sequences were identical to species B (HAdV-3) and one sequence to species E (HAdV-4). Furthermore, a phylogenetic tree was constructed (Figure) using the sequences obtained in this study and 
27 different HAdV reference sequences (Allard et al. 2001) obtained from GenBank (http://www.ncbi.nlm. nih.gov/Genbank), which corroborated the results obtained using BLAST.

A different circulation pattern was observed in São Paulo by Moura et al. (2007) in samples collected between March 1995-March 1996 and between April 2000-April 2001. The most prevalent serotype from this study was HAdV-7 (56.7\%), followed by HAdV-1 (26.7\%), HAdV-3 (6.7\%), HAdV-2, HAdV-5 and HAdV-6 (3.3\% each). However, in that study samples were only collected from hospitalized children. Our study included samples collected from children who presented ARD at the Paediatric Emergency Service, the Paediatric Ward, the Paediatric Intensive Care Unit and the Neonatal Intensive Care Unit.

In this study, a characterization into serotypes was also performed in silico. This was accomplished by determining the presence of restriction sites listed in the flowchart proposed by Allard et al. (2001). Four specimens of

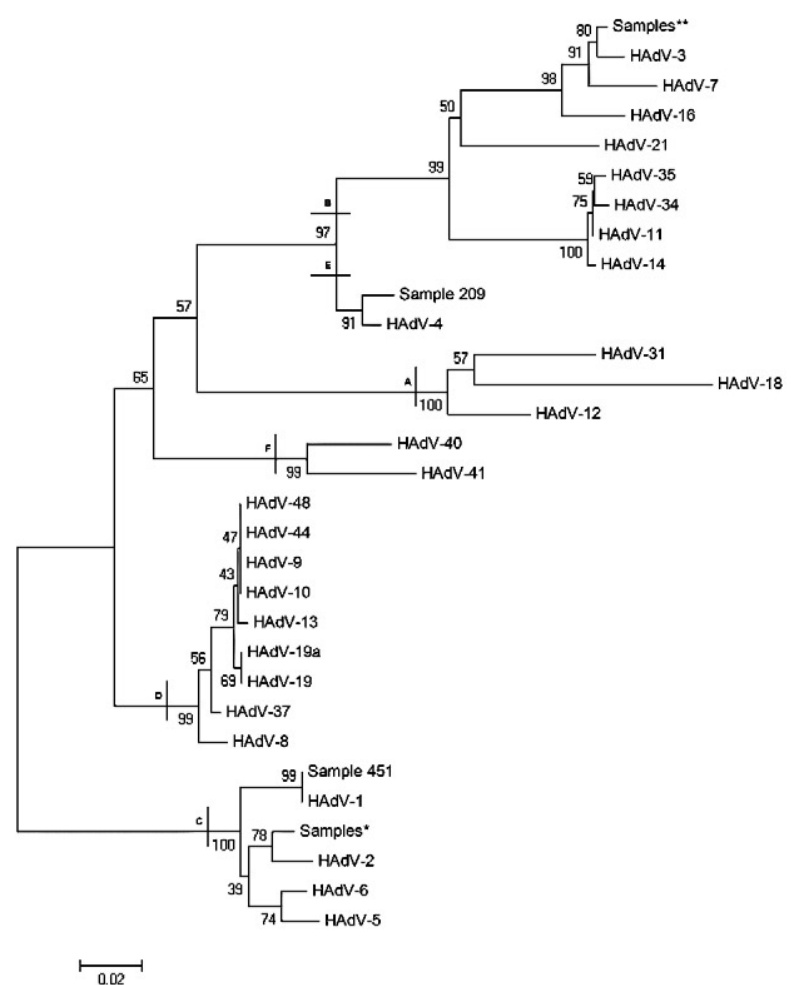

Phylogenetic tree of nucleotide sequences of clinical specimens with 253 bp sequence within the adenovirus hexon gene. Bootstrap values (from 1,000 replicates) are indicated as a percentage in each node. The following published sequences were used: HAdV1 (AF161559), HAdV2 (J01917), HAdV3 (X76549), HAdV5 (X02997), HAdV4 (AF161569), HAdV6 (AF161560), HAdV7 (X76551), HAdV8 (AF161561), HAdV9 (AF161562), HAdV10 (AF161563), HAdV11 (AF161570), HAdV12 (X73487), HAdV13 (AF161564), HAdV14 (AF161571), HAdV16 (X74662), HAdV18 (AF161575), HAdV19 (AF161565), HAdV19a (AF161566), HAdV21 (AF161572), HAdV31 (AF161576), HAdV34 (AF161573), HAdV35 (AF161574), HAdV37 (AF161567), HAdV40 (X51782), HAdV41 (X51783), HAdV44 (AF161568) and HAdV48 (U20821).
HAdV-3 belonged to the type $3 p$ genome. This serotype was previously observed in patients with either respiratory disease or conjunctivitis in the cities of Belém (North Brazil) and Rio de Janeiro (Southeast) (Kajon et al. 1999).

Through nucleotide sequence analysis, we determined that eight specimens were from species C. Due to the limitations of the protocol proposed by Allard et al. (2001), caused by the lack of other restriction sites in the amplicons, the serotypes of seven specimens could not be determined and might be HAdV-2, HAdV-5 or HAdV-6. However, a BLAST search showed the highest identity to HAdV-2. Nevertheless, the presence and absence of certain restriction sites in their nucleotide sequences confirmed the results obtained in the phylogenetic analysis and BLAST search.

Although serotype HAdV-4 is frequently associated with ARD in military recruits (Kolavic-Gray et al. 2002), one specimen of this serotype was identified in a twoyear-old child. The nucleotide sequence was $100 \%$ identical to the HAdV-4 isolated from basic training recruits in United States of America during the end of the 1990s (Blasiole et al. 2004). This serotype was also found in Japan, where it was associated with cases of conjunctivitis (Miura-Ochiai et al. 2007). To our knowledge, this is the first description of serotype HAdV-4 in Brazil.

In addition, we found the nucleotide sequence of specimen AdV-UDI-451 to be $100 \%$ identical to two feline AdV sequences (Lakatos et al. 1999, and data reported in GeneBank accession AY512566). One of these sequences was detected in a cat that suffered from a transient hepatic failure (Lakatos et al. 1999). A similar result was found by Phan et al. (2006), who reported that the amino acid sequence of the AdV hexon gene, obtained from the stool specimen of a one-year-old child with gastroenteritis in Japan, grouped together with the feline adenovirus sequence AY512566 in a phylogenetic analysis, suggesting that HAdV-1 may be transmitted from felines to humans and/or vice versa. However, a cat was not kept at the family's house and it was not clear whether there was contact with the animals that lived in the surrounding area. In our study, we did not determine whether the family of the HAdV-1-infected child had a cat.

In conclusion, using a combination of IF and PCR methodologies allowed us to determine a $7.1 \%$ prevalence rate of HAdV infection in clinical samples from children presenting with ARDs in Uberlândia. Furthermore, HAdV-2 was the most prevalent serotype found among the HAdV specimens. HAdV-4, which had not been previously described to circulate in Brazil and an isolate with strong similarity to the feline AdV were also found.

\section{ACKNOWLEDGEMENTS}

To Dr. Dean D Erdman, Centers for Disease Control and Prevention, and to Dr. Marilda Siqueira, Laboratório de Vírus Respiratórios e Sarampo, IOC-Fiocruz, for providing the Respiratory Panel I Viral Screening and Identification kit, and to the laboratories of Immunology, Parasitology, Molecular Biology, Physiology and Genetics, UFU, to permit us to use their facilities and the health care professionals of the Clinics Hospital of Uberlândia for the screening of patients. 


\section{REFERENCES}

Allard A, Albinsson B, Wadell G 2001. Rapid typing of human adenoviruses by a general PCR combined with restriction endonuclease analysis. J Clin Microbiol 39: 498-505.

Araújo AA, Yokosawa J, Durigon EL, Ventura AM 2001. Polymerase chain reaction detection of adenovirus DNA sequences in human lymphocytes. Braz J Microbiol 32: 153-157.

Blasiole DA, Metzgar D, Daum LT, Ryan MA, Wu J, Wills C, Le CT, Freed NE, Hansen CJ, Gray GC, Russell KL 2004. Molecular analysis of adenovirus isolates from vaccinated and unvaccinated young adults. $J$ Clin Microbiol 42: 1686-1693.

Brandt CD, Kim HW, Vargosko AJ, Jeffries BC, Arrobio JO, Rindge B, Parrott RH, Chanock RM 1969. Infections in 18,000 infants and children in a controlled study of respiratory tract disease. I. Adenovirus pathogenicity in relation to serologic type and illness syndrome. Am J Epidemiol 90: 484-500.

Cabello C, Manjarrez ME, Olvera R, Villalba J, Valle L, Paramo I 2006. Frequency of viruses associated with acute respiratory infections in children younger than five years of age at a locality of Mexico City. Mem Inst Oswaldo Cruz 101: 21-24.

Costa LF, Yokosawa J, Mantese OC, Oliveira TFM, Silveira HL, Nepomuceno LL, Moreira LS, Dyonisio G, Rossi LMG, Oliveira RC, Ribeiro LZG, Queiróz DAO 2006. Respiratory viruses in children younger than five years old with acute respiratory disease from 2001 to 2004 in Uberlândia, MG, Brazil. Mem Inst Oswaldo Cruz 101: 301-306.

de Arruda E, Hayden FG, McAuliffe JF, de Sousa MA, Mota SB, McAuliffe MI, Geist FC, Carvalho EP, Fernandes MC, Guerrant RL, Gwaltney JM Jr 1991. Acute respiratory viral infections in ambulatory children of urban Northeast Brazil. J Infect Dis 164: 252-258.

Gray GC, Chorazy ML 2009. Human adenovirus 14a: a new epidemic threat. J Infect Dis 199: 1413-1415.

Hall TA 1999. BioEdit: a user-friendly biological sequence alignment editor and analysis program for Windows 95/98/NT. Nucleic Acids Symp Ser 41: 95-98.

Hong JY, Lee HJ, Piedra PA, Choi EH, Park KH, Koh YY, Kim WS 2001. Lower respiratory tract infections due to adenovirus in hospitalized Korean children: epidemiology, clinical features and prognosis. Clin Infect Dis 32: 1423-1429.

Horwitz MS 2001. Adenoviruses. In DM Knipe, PM Howley (eds.), Fields virology, 4th ed., Lippincott Williams \& Wilkins, Philadelphia, p. 2301-2326.

Ishiko H, Aoki K 2009. Spread of epidemic keratoconjunctivitis due to a novel serotype of human adenovirus in Japan. J Clin Microbiol 47: 2678-2679.

Jones MS 2nd, Harrach B, Ganac RD, Gozum MM, Dela Cruz WP, Riedel B, Pan C, Delwart EL, Schnurr DP 2007. New adenovirus species found in a patient presenting with gastroenteritis. J Virol 81: 5978-5984.

Kajon AE, Portes SA, de Mello WA, Nascimento JP, Siqueira MM 1999. Genome type analysis of Brazilian adenovirus strains of serotypes 1, 2, 3, 5 and 7 collected between 1976 and 1995. $J$ Med Virol 58: 408-412.

Kolavic-Gray SA, Binn LN, Sanchez JL, Cersovsky SB, Polyak CS, Mitchell-Raymundo F, Asher LV, Vaughn DW, Feighner BH, Innis BL 2002. Large epidemic of adenovirus type 4 infection among military trainees: epidemiological, clinical, and laboratory studies. Clin Infect Dis 35: 808-818.
Kumar S, Tamura K, Nei M 2004. MEGA3: integrated software for molecular evolutionary genetics analysis and sequence alignment. Brief Bioinform 5: 150-163.

Lakatos B, Farkas J, Egberink HF, Vennema H, Horzinek MC, Benkó M 1999. Detection of adenovirus hexon sequence in a cat by polymerase chain reaction (short communication). Acta Vet Hung 47: 493-497.

Larrañaga C, Martínez HJ, Palomino MA, Peña CM, Carrión AF, Avendaño CLF 2007. Molecular characterization of hospital-acquired adenovirus infantile respiratory infection in Chile using species-specific PCR assays. J Clin Virol 39: 175-181.

Lewis PF, Schmidt MA, Lu X, Erdman DD, Campbell M, Thomas A, Cieslak PR, Grenz LD, Tsaknardis L, Gleaves C, Kendall B, Gilbert D 2009. A community-based outbreak of severe respiratory illness caused by human adenovirus serotype 14 . J Infect Dis 199: 1427-1434.

Miura-Ochiai R, Shimada Y, Konno T, Yamazaki S, Aoki K, Ohno S, Suzuki E, Ishiko H 2007. Quantitative detection and rapid identification of human adenoviruses. J Clin Microbiol 45: 958-967.

Moura PO, Roberto AF, Hein N, Baldacci E, Vieira SE, Ejzenberg B, Perrini P, Stewien KE, Durigon EL, Mehnert DU, Hársi CM 2007. Molecular epidemiology of human adenovirus isolated from children hospitalized with acute respiratory infection in São Paulo, Brazil. J Med Virol 79: 174-181.

Murtagh P, Giubergia V, Viale D, Bauer G, Pena HG 2009. Lower respiratory infections by adenovirus in children. Clinical features and risk factors for bronchiolitis obliterans and mortality. Pediatr Pulmonol 44: 450-456.

Nascimento JP, Siqueira MM, Sutmoller F, Krawczuk MM, de Farias V, Ferreira V, Rodrigues MJ 1991. Longitudinal study of acute respiratory diseases in Rio de Janeiro: occurrence of respiratory viruses during four consecutive years. Rev Inst Med Trop Sao Paulo 33: 287-296.

Oliveira TFM, Freitas GRO, Ribeiro LZG, Yokosawa J, Siqueira MM, Portes SAR, Silveira HL, Calegari T, Costa LF, Mantese OC, Queiróz DAO 2008. Prevalence and clinical aspects of respiratory syncytial virus $A$ and $B$ groups in children seen at Hospital de Clínicas of Uberlândia, MG, Brazil. Mem Inst Oswaldo Cruz 103: 417-422.

Phan TG, Shimizu H, Nishimura S, Okitsu S, Maneekarn N, Ushijima H 2006. Human adenovirus type 1 related to feline adenovirus: evidence of interspecies transmission. Clin Lab 52: 515-518.

Queiróz DA, Durigon EL, Botosso VF, Ejzemberg B, Vieira SE, Mineo JR, Yamashita C, Hein N, Lopes CL, Cacharo AL, Stewien KE 2002. Immune response to respiratory syncytial virus in young Brazilian children. Braz J Med Biol Res 35: 1183-1193.

Rosete DP, Manjarrez ME, Barrón BL 2008. Adenoviruses C in nonhospitalized Mexican children older than five years of age with acute respiratory infection. Mem Inst Oswaldo Cruz 103: 195-200.

Sarantis H, Johnson G, Brown M, Petric M, Tellier R 2004. Comprehensive detection and serotyping of human adenoviruses by PCR and sequencing. J Clin Microbiol 42: 3963-3969.

Straliotto SM, Siqueira MM, Muller RL, Fischer GB, Cunha ML, Nestor SM 2002. Viral etiology of acute respiratory infections among children in Porto Alegre, RS, Brazil. Rev Soc Bras Med Trop 35: 283-291.

Swenson PD, Wadell G, Allard A, Hierholzer JC 2003. Adenoviruses. In PR Murray, EJ Baron, JH Jorgensen, MA Pfaller, RH Yolken 
(eds.), Manual of clinical microbiology, 8th ed., American Society for Microbiology, Washington, p. 1404-1417.

Tate JE, Bunning ML, Lott L, Lu X, Su J, Metzgar D, Brosch L, Panozzo CA, Marconi VC, Faix DJ, Prill M, Johnson B, Erdman DD, Fonseca V, Anderson LJ, Widdowson MA 2009. Outbreak of severe respiratory disease associated with emergent human adenovirus serotype 14 at a US air force training facility in 2007. J Infect Dis 199: 1419-1426.

Tsuchiya LR, Costa LM, Raboni SM, Nogueira MB, Pereira LA, Rotta I, Takahashi GR, Coelho M, Siqueira MM 2005. Viral respiratory infection in Curitiba, Southern Brazil. J Infect 51: 401-407.

Vieira SE, Stewien KE, Queiroz DA, Durigon EL, Török TJ, Anderson LJ, Miyao CR, Hein N, Botosso VF, Pahl MM, Gilio AE, Ejzenberg B, Okay Y 2001. Clinical patterns and seasonal trends in respiratory syncytial virus hospitalizations in São Paulo, Brazil. Rev Inst Med Trop Sao Paulo 43: 125-131.

Walsh MP, Chintakuntlawar A, Robinson CM, Madisch I, Harrach B, Hudson NR, Schnurr D, Heim A, Chodosh J, Seto D, Jones MS 2009. Evidence of molecular evolution driven by recombination events influencing tropism in a novel human adenovirus that causes epidemic keratoconjunctivitis. PLoS One 4: e5635.

Williams BG, Gouws E, Boschi-Pinto C, Bryce J, Dye C 2002. Estimates of world-wide distribution of child deaths from acute respiratory infections. Lancet Infect Dis 2: 25-32.

Wood SR, Sharp IR, Caul EO, Paul I, Bailey AS, Hawkins M, Pugh S, Treharne J, Stevenson S 1997. Rapid detection and serotyping of adenovirus by direct immunofluorescence. J Med Virol 51: 198-201. 